\title{
Ökonomisierung aus organisationssoziologischer Perspektive: Der Beitrag der Medienunternehmen zur Ökonomisierung
}

\author{
Klaus-Dieter Altmeppen
}

Im Mittelpunkt dieses Beitrags stebt eine Analyse der Ökonomisierung auf der Ebene von Medienunternebmen. Als Organisationen sind Medienunternebmen vor allem durch Ziele und Strukturen charakterisiert, wobei sie neben dem Profitprinzip auch publizistische Ziele verfolgen und beide Ziele in Einklang bringen müssen. Da die Ziele Entscheidungsprämissen der Medien darstellen, kann eine Ökonomisierung auf organisationaler Ebene dann konstatiert werden, wenn wirtschaftliche Ziele die publizistischen Entscheidungen zunebmend dominieren. Anhand von fünf Thesen wird geprüft, ob die Ökonomisierung des Mediensystems auch auf der Organisationsebene festgestellt werden kann. Thematisiert werden die Ökonomisierung der Entscheidungsprämissen und somit der Strategien der Medienunternehmen, die Ökonomisierung der Planungs- und Produktionsprozesse und schließlich die Ökonomisierung des Angebots. Im Ergebnis kann von einer Spirale der Ökonomisierung („Ökonomisierung der Ökonomisierung “) gesprochen werden. Aufgrund rekursiver Regulierung und Strukturierung treiben die Medienunternebmen die Ökonomisierung aktiv und mit machtvollen Mitteln voran.

\section{1. Ökonomisierung und Kommerzialisierung: Eine Begriffsbestimmung aus organisationssoziologischer Perspektive}

Ökonomisierung ist kein eindimensionales Phänomen und so kann der Prozess der Ökonomisierung auf mehreren Ebenen erfasst werden. Auf der Makroebene wird Ökonomisierung als ein Prozess der De-Regulierung und Liberalisierung von Märkten und Wettbewerb angesehen. Eine zweite Form der Ökonomisierung entsteht durch das Handeln von Akteuren oder Akteursgruppen in den Medienunternehmen, im Mesobereich avancieren folglich die Medienunternehmen zum Untersuchungsobjekt.

Medien können, fasst man sie als erwerbswirtschaftliche Einheiten mit Profitstreben, als Unternehmen im Rahmen der Institutionenökonomie oder als Organisationen im Rahmen der Organisationssoziologie analysiert werden. Als ökonomische Institutionen werden Medienunternehmen in ihren Wechselwirkungen mit Markt und Wettbewerb thematisiert (vgl. Kiefer 2001, 72 ff.), dabei stehen die vertraglichen und transaktionsgebundenen Handlungen der Unternehmen im Vordergrund. Das Interesse der Organisationssoziologie richtet sich stärker auf Verschränkungen zwischen den Strukturen, die in der Regel als Aufbauorganisation bezeichnet werden, und den Handlungsprozessen, also der Prozessgestaltung in Form der Ablauforganisation (vgl. von der Oelsnitz 2000; Krüger 1994; Hill/Fehlbaum/Ulrich 1994). Dabei schält sich ein Zweig heraus, dessen Erkenntnisinteresse darauf gerichtet ist, die rekursiven Verschränkungen von Handeln und Strukturen zu fokussieren (vgl. Ortmann/Sydow/Windeler 1997). Diese Theorietradition lehnt sich in starkem Maß an die Strukturationstheorie von Giddens (1997) an, um ein tragfähiges Fundament für die Möglichkeiten und Restriktionen von Unternehmen und Akteuren zu schaffen.

Der Mehrwert strukturationstheoretischer Verortung von Medienunternehmen liegt vor allem darin, den aktiven Part von Unternehmen an den ökonomischen Erscheinun- 
gen hervorzuheben. Nicht allein Märkte sind danach die bestimmenden Faktoren des Mediensystems, sondern die Unternehmen regulieren diese Märkte aktiv und schaffen damit Marktstrukturen - und die Unternehmen werden von den Märkten reguliert und müssen ihre internen Strukturen und ihre Strategien darauf einrichten (vgl. Altmeppen 2001).

Im Folgenden soll die Ökonomisierung unter Rückgriff auf diese organisationssoziologischen Ansätze analysiert werden. Auf der Mesoebene setzt sich immer mehr die Ansicht durch, Kommerzialisierung als einen Aspekt der Ökonomisierung anzusehen, da der Begriff Kommerzialisierung insbesondere auf die Folgen überbordender ökonomischer Prinzipien auf der Unternehmensebene abzielt (vgl. McQuail 1986). Kommerzialisierung auf Unternehmensebene wäre dann als „Zurückdrängen der gesellschaftlich erwünschten meritorischen zu Gunsten der auf Märken verkäuflichen und einzelwirtschaftlich rentablen Angebote" (Kiefer 2001, 22) zu verstehen.

Ökonomisierung (der Begriff soll im Rahmen dieses Beitrags beibehalten werden) findet also auf der Ebene von Organisationen als ein geplanter - wenn auch in den Folgen nicht immer intendierter -, einem rekursiven Strukturierungs-/Regulierungskonzept folgender Prozess statt, bei dem für alle Medienorganisationen zunehmend die ökonomischen Regeln kapitalistischer Gesellschaften gelten und bei dem publizistisches Handeln weitgehend von wirtschaftlichen Kalkülen geprägt wird.

Es geht bei der Ökonomisierung nicht um die Frage, ob dies ein neues Phänomen ist. Medienunternehmen handeln grundsätzlich medienwirtschaftlich, denn für Medienunternehmen - auch für öffentlich-rechtliche - ist die Geldabhängigkeit konstitutiv, also die Beschaffung, Bewirtschaftung und Mehrung des Geldes. Die gegenwärtigen Prozesse der Ökonomisierung verweisen aber auf eine Phase besonders intensiver Dominanz ökonomischen Kalküls.

So ist die Tatsache, dass es Werbung gibt, kein Beleg für die Ökonomisierung. Ein Beleg ist aber sehr wohl, dass die Medienunternehmen die Werbung ausdehnen (durch Steigerung der Werbezeiten), intensivieren (durch Erhöhung der Werbeetats), verfeinern (durch Marktforschung) und konzentrieren (durch Fusionen der Werbeanbieter).

Aufgrund dieser Ökonomisierungstendenzen stellt sich die Frage, worauf die Dominanz der Ökonomisierung beruht, wo ihre Ursachen auf der Unternehmensebene festgemacht werden können und welche Merkmale für eine Kommerzialisierung der Medienunternehmen charakteristisch sind. Mit den folgenden Thesen ist daher der Versuch verbunden, Phänomene der Ökonomisierung aufgrund theoriegeleiteter Hypothesen auf organisationaler Ebene zu identifizieren. Zu Beginn sollen zuerst die Merkmale von Medienunternehmen als Organisationen aufgezeigt werden.

\section{Medienunternehmen als Organisationen - eine begriffliche Konkretisierung}

Medienunternehmen können als Systeme organisierten Handelns aufgefasst werden, die kommunikationsabhängig (Organisationen konstituieren sich über Kommunikation) sowie entscheidungsfixiert (Entscheidungen sind die zentralen Operationsweisen) und ressourcengebunden (Geld) sind.

Medienunternehmen sind Leistungsorganisationen des Wirtschaftssystems und agieren dementsprechend nach wirtschaftlichen Kriterien. Als Wirtschaftsunternehmen handeln sie nach der Devise von Zahlung/Nichtzahlung, nicht nach dem Code von öffentlich/nicht-öffentlich, der für den Journalismus die zentrale Handlungsleitung ist. Nicht die Veröffentlichungen, sondern die über Markthandlungen erfolgenden Zahlungen entscheiden über den Fortbestand und die künftige Entwicklung der Medienunternehmen. 
$\mathrm{Zu}$ den zentralen organisationalen Charakteristika gehören die Ziele und Strukturen der Medienunternehmen. Üblicherweise gelten das autonome Wirtschaften, das Profitprinzip und das Privateigentum der Produktionsmittel als Kennzeichen von Unternehmen. Im Gegensatz zu den meisten anderen Unternehmen müssen Medienunternehmen jedoch neben dem Profitprinzip auch publizistische Ziele verfolgen und beide Ziele in Einklang bringen. Da sie sowohl auf dem Rezipienten- wie auf dem Werbemarkt erfolgreich sein müssen, spiegeln sich die Mechanismen des Verbundproduktes „Medien“ (vgl. Kiefer 2001, 151 ff.) auch in den dichotomen Zielen wider.

Ziele repräsentieren den Sinn von Organisationen, mit den Zielen werden die Operationen in den Medien strukturiert. Ziele stellen in diesem Sinn die Entscheidungsprämissen der Medien dar. Zu diesen Grundlagen des medienunternehmerischen Handelns gehören die Geschäftsziele sowie die unternehmerischen Strategien auf der einen und die publizistischen Ziele auf der anderen Seite. In sozialer Hinsicht werden die Mitarbeiter auf die Entscheidungsprämissen verpflichtet und zu deren Umsetzung angehalten. Entscheidungsprämissen regulieren als Sollensebene die Regeln und Verfahren, nach denen Medienangebote produziert werden. In sachlicher Hinsicht legen die Ziele die organisationalen Strukturierungen fest, in zeitlicher Hinsicht regulieren Ziele die Ereigniszeitpunkte (zum Beispiel die Einführung neuer Zeitschriften oder Programmformate). Im Hinblick auf die Ziele der Medienunternehmen ist eine Ökonomisierung in erster Linie dann zu konstatieren, wenn wirtschaftliche Ziele die publizistischen Entscheidungen zunehmend dominieren (vgl. Altmeppen 2000).

Analog zu den Zielen bilden Medienunternehmen ihre organisationale Gestaltung aus. Die Strukturen werden aus den Zielen abgeleitet und bestehen aus den Regeln, den Verfahrensweisen und Ressourcen, denn nachdem „die organisatorischen Zielkriterien definiert sind, stellt sich als zweites die Aufgabe, organisatorische Instrumentalvariablen zu finden, durch deren Einsatz organisatorische Ziele unter gegebenen Constraints realisiert werden können.“ (Hill/Fehlbaum/Ulrich 1994, 170)

Die „Instrumentalvariablen“ der Medienunternehmen bestehen aufbauorganisatorisch typischerweise in der Einrichtung von Ressorts und Programmredaktionen sowie den Abteilungen für Werbung und Vertrieb (vgl. Weischenberg 1992, 275ff.) und ablauforganisatorisch in einer entsprechenden Organisation der Geschäfts- und Produktionsprozesse nach Verantwortlichkeiten und Zuständigkeiten (vgl. von der Oelsnitz 2000, 113; Krüger 1994, 15 ff.). Dies bedeutet einerseits, dass Medienunternehmen die Regeln und Ressourcen ihrer Ablauf- und Aufbauorganisation (die Strukturen) zielorientiert planen, steuern und kontrollieren (vgl. von der Oelsnitz 2000). In diese Strukturen ist das publizistische und ökonomische Handeln der Medienschaffenden eingebettet. Die Strukturen ermöglichen erfolgreiches publizistisches Handeln und schränken es zugleich ein, denn die zeitlichen Abläufe der aktuellen Medienproduktion beispielsweise sind ohne konkrete Regeln und Verfahren kaum zu bewältigen. Die aufbau- und ablauforganisatorischen Instrumente und Verfahrensweisen der Medienunternehmen können als Entscheidungsprogramme bezeichnet werden. Mit den Entscheidungsprogrammen werden die Ziele der Medienunternehmen insoweit konkretisiert, als sie Erwartungen an das publizistisch-ökonomische Handeln der Organisationsmitglieder formulieren (vgl. Luhmann 1987, 278 f.; 432 f.).

Die Ermöglichung und Einschränkung medienwirtschaftlichen Handelns resultiert aus den situativen Bedingungen, den Constraints. Inwieweit die Entscheidungsprogramme - und damit im weiteren die Organisationsziele - erfolgreich umgesetzt werden können, entscheidet sich über die situativen Bedingungen. Hierzu gehören zum Beispiel 
das Vorhandensein oder Fehlen von Informationen, die Konkurrenzbedingungen und die Möglichkeit, Ressourcen zu aktivieren.

Die Verfahrensweisen und Regeln in den Medienunternehmen werden also nicht einfach festgesetzt und dann fortgeschrieben, sondern sie werden auch verändert, weil sie den Umweltbedingungen und den situativen Anforderungen angepasst werden (vgl. Ortmann/Sydow/Windeler 1997, 318 ff.). Insbesondere die Marktorientierung der Medienunternehmen (vgl. von der Oelsnitz 2000, 13 ff.) fordert permanent dazu heraus, die Entscheidungsprämissen und die operativen Entscheidungsprogramme zu vergleichen und möglicherweise anzupassen. Dem medienwirtschaftlichen Handeln ist somit eine Rekursivität von Regulierungen und Strukturierungen inhärent. Rekursive Regulierung und Strukturierung bedeutet, dass die Strategie Grundlage, aber auch Folge der (Markt-)Strukturen sein kann. Grundlage ist sie, wenn durch Strategien Marktstrukturen geschaffen oder verändert werden. Folge ist sie, wenn Strategien aufgrund von Marktstrukturen verändert werden (vgl. Ortmann/Sydow/Windeler 1997, 346 ff.; Zimmer/Ortmann 1996, 90). Rekursive Regulierung und Strukturierung bedeutet weiterhin, dass „gerade dadurch, daß Regulation es an sich hat, das Handlungsfeld von Unternehmen zu strukturieren - also: zu öffnen und zu verengen -, ein strategisches Interesse und ein strategisches Bemühen dieser Unternehmungen hervorgerufen wird, ihrerseits auf die sie betreffenden Regulationen Einfluß zu nehmen." (Zimmer/Ortmann 1996, 105) Den Wettbewerb mit seinen permanent wandelnden Strategien und Strukturen empfinden die Konkurrenten als Regulierung, die sie strategisch beeinflussen und aktiv gestalten wollen.

Die Rekursivität ergibt sich insbesondere aus den Unsicherheiten und Risiken der Medienproduktion. So können im aktuellen Nachrichtenjournalismus Berichterstattungsereignisse häufig nicht geplant werden, denn Ereignisse sind nur selten planbar. Andererseits ist auch der Erfolg neuer Fernsehformate nicht prognostizierbar. Daher müssen die Strategien der Medienunternehmen immer wieder an die veränderten Umwelterfordernisse angepasst werden. Die Schleifen zwischen den internen Organisationsstrukturen und den Umweltdynamiken verlaufen rekursiv: Umweltveränderungen können innerorganisationale Restrukturierungen auslösen, wenn beispielsweise der Kirch-Konzern umfassende Organisationsveränderungen vornimmt, um den Konzern an die neuen Geschäftsmodelle des Pay-TV anzupassen (vgl. Röper 2001, 2). Andererseits wirken gerade diese organisationalen Veränderungen auf die Märkte zurück, denn die Konkurrenten passen ihre Strategien ebenfalls an die neuen Gegebenheiten an.

Wenn nun Medienorganisationen auf rekursive Weise ihre Ziele und Strukturen verändern, wenn sie Regeln und Produktionsprozesse anpassen, wenn sie ihre Ressourcen verlagern und an gewandelte Kundenbedürfnisse anpassen, werden diese Veränderungen beobachtbar und können möglicherweise als Merkmale der Ökonomisierung gedeutet werden. Diese Prozesse der Anpassung von publizistisch-ökonomischem Handeln an Strukturen und der Veränderung von Strukturen durch publizistisches Handeln sollen im Folgenden thesenhaft illustriert werden.

\section{3. Ökonomisierung der Entscheidungsprämissen}

Als erste These kann festgehalten werden, dass eine Ökonomisierung der Entscheidungsprämissen und somit der Strategien der Medienunternehmen beobachtet werden kann. Mit den Entscheidungsprämissen formulieren die Unternehmen die Rahmenbedingungen, die publizistischen und die ökonomischen Ziele. In der Strategielehre werden die möglichen Ziele nach innovativ und reaktiv, nach Kostenführerschaft und Pro- 
duktdifferenzierung sowie nach internem und externem Wachstum unterschieden (vgl. Sjurts 1996, 5). Strategien in Medienmärkten können jedoch immer weniger langfristig festgelegt werden. Um auf den Medienmärkten erfolgreich zu agieren, müssen Medienunternehmen permanent Entscheidungen über ihr Marktverhalten treffen und ihre Strategien an veränderte Marktstrukturen anpassen.

Entscheidungsprämissen von Medienunternehmen bewegen sich somit immer auf einem schmalen Grat von publizistischem vs. ökonomischem Erfolg. Beide Erfolgsvarianten können durchaus deckungsgleich sein, wie etwa das Beispiel der Wirtschaftspresse verdeutlicht. Der Ausbau dieses Marktsegments, das grundsätzlich eine hohe Nachfrageflexibilität aufweist (vgl. Sjurts 1996, 71), beruht auf der gesteigerten Nachfrage, in dessen Folge sowohl neue Titel aufgelegt wie auch bestehende Titel ausgebaut wurden.

Publizistisch induzierte Entscheidungsprämissen werden jedoch mehr und mehr zur Ausnahme. Ein zentrales Merkmal der Ökonomisierung liegt somit darin, dass die Entscheidungsprämissen und Strategien der Medienunternehmen im Blick auf ökonomisch Erfolg versprechende Kriterien ausgerichtet werden, wenn also das Unternehmensergebnis als Maßstab des Erfolges in den Vordergrund rückt und publizistische Entscheidungsprämissen verdrängt.

So wird in der Programmplanung der TV-Sender beispielsweise deutlich, dass die Sendeinhalte zeitlich und inhaltlich nach Markterfolgskriterien gestaltet werden. Die Entwicklung neuer Formen und Formate (wie Populärjournalismus und InvolvementTV) resultiert aus differenzierter Marktbeobachtung und Marktforschung, deren Kriterien nicht aus dem Sachziel publizistischer Wettbewerb resultieren, sondern aus ökonomischen Gründen. Dies zeigt sich am hohen Anteil imitativer statt innovativer Produktneuerungen. Innovative Neuschöpfungen im Zeitschriftenmarkt, die maßstabsgetreue Adaption bestehender Zeitungstitel im WWW, neue Formate im TV: Immer folgen den innovativen und erfolgreichen Erstlingen regelhaft Imitationen, deren Ziel nicht in erster Linie darin liegt, ein Qualitäts- oder Alleinstellungsmerkmal zu schaffen, sondern am Erfolg zu partizipieren, Nischen zu besetzen und Monopole zu verhindern.

$\mathrm{Zu}$ den Entscheidungsprämissen, die derzeit in Medienmärkten dominieren, gehören des Weiteren die Strategien zum Wettbewerb. Konkurrenzverhältnisse werden im Medienbereich zunehmend von strategischen Allianzen (vgl. Altmeppen 1996) und Großfusionen geprägt. Die Medienunternehmen entscheiden nicht alternativ zwischen internem und externem Wachstum, sondern sie entscheiden anhand der Opportunität in den jeweiligen Situationen, in welchem Maße Wachstum erfolgen wird. Auch der Bertelsmann-Konzern verfolgt keine einheitliche Strategie: Im TV-Bereich dominiert eher externes Wachstum, wie die Fusion der CLT-UFA mit der britischen Pearson-Gruppe zeigt (vgl. Röper 2001, 2f.), während die Konzerntochter Gruner+Jahr stärker auf internes Wachstum setzt (vgl. Sjurts 1996, 108). Ein weiterer Beleg für die Ökonomisierung der Entscheidungsprämissen zeigt sich im - aus Wettbewerbsperspektive wenig beachteten - Werbesektor. Mit Kirch und Bertelsmann haben sich nicht nur Senderfamilien etabliert, die jeden weiteren Marktzutritt verhindern können, die beiden Konzerne haben zudem auch die Werbevermarkter konzentriert und in die Senderfamilien eingebunden (vgl. Horizont 27/2000, 6.7.2000, 45). Die Entscheidungsprämissen lauten hier, durch die Senderfamilien die Wertschöpfungskette zu sichern und durch die Konzentration der Werbepartner eine optimale Vermarktung zu garantieren.

Wenn nun die Entscheidungsprämissen und Strategien ökonomisiert werden, dann hat das auch Folgen für die Entscheidungsprogramme, also die Planungs- und Produktionsprozesse in den einzelnen Unternehmensteilen. 


\section{4. Ökonomisierung der Entscheidungsprogramme}

Ökonomisierung, so die zweite These, bedeutet auch die verstärkte Ausrichtung der Entscheidungsprogramme - und damit der Planungs- und Produktionsprozesse - an wirtschaftlichen Kriterien. Begleitet werden diese Veränderungen vorrangig von der Hoffnung auf Kostenminimierung und einem verschärften Kostencontrolling.

Gruner + Jahr beispielsweise hat alle Zeitschriftentitel in Profitcentern zusammengefasst, an deren Spitze ein Verlagsgeschäftsführer steht (vgl. Horizont 26/2000, 29.6.2000, 50). Auf diese Weise soll der Zeitschriftenbereich auf der Managementebene für die Zukunft fit gemacht werden, da die Profitcenter nach dem Markterfolg bewertet und gesteuert werden (vgl. zu Profitcentern allgemein: von der Oelsnitz 2000, 53 ff.). Der Schub an Ökonomisierung, der von Profitcentern ausgeht, resultiert unter anderem daraus, dass in Profitcentern - die in einem unternehmensinternen Wettbewerb stehen kurzfristige Erfolge und übersteigertes Gewinnstreben vor langfristiger Unternehmenssicherung und innovativer Produktpolitik dominieren (vgl. von der Oelsnitz 2000, 57).

Das Kosten- und Erfolgsprinzip führt auf der Ebene der Produktion - Redaktionen, Produzenten - dazu, die Produktionsprozesse in höherer Form als in der Vergangenheit an ökonomischen Prinzipien zu orientieren. Die Effektivierung von Produktionsprozessen führt beispielsweise bei Tageszeitungen und im Hörfunk dazu, die Ressorts aufzulösen. Statt dieser thematischen Gliederung der Aufbauorganisation werden großflächige Bereiche eingerichtet, in denen die Journalistinnen und Journalisten neben der inhaltlichen Bearbeitung auch für die druckfertigen Vorlagen bzw. die Einhaltung der Formate zuständig sind (vgl. Moss 1998; Altmeppen/Donges/Engels 1999, 266). In zunehmendem Maße bestehen die Anforderungen für die Redaktionen darin, ein effizientes - also kostenorientiertes - Redaktionsmanagement einzurichten und ein gesamtunternehmerisches Marketing zu betreiben (vgl. Altmeppen 1999, 183 ff.). Insgesamt werden die Redaktionen nicht nur zu mehr unternehmerischem Denken angehalten, sondern strukturell (durch Zielsetzungen, Regeln und Ressourcen) ökonomisiert. Da die Redaktionsorganisation ein Spiegel des publizistischen Ziels von Medienunternehmen ist, deuten der Rückgang differenzierter redaktioneller Organisation zugunsten großflächiger Bereiche und die Strukturierungen im Hinblick auf wirtschaftlichen Erfolg auf einen Vorrang ökonomischer Entscheidungsprogramme vor publizistischen Operationen.

\section{5. Ökonomisierung der Ressourcenallokation}

Ressourcen stellen in mehrfacher Hinsicht einen bedeutsamen Teil medienunternehmerischer Operationen dar. Zum einen sind allokative Ressourcen - wie Finanzmittel, Güter, Rechte - konstitutiver Bestandteil des unternehmerischen Handelns. Zum anderen stellen autoritative Ressourcen, wie etwa Kernkompetenzen (herausragende Fähigkeiten eines Unternehmens und strategische Vermögenswerte wie Reputation), wichtige Kriterien für dauerhafte Wettbewerbsvorteile und Gewinn- oder Renditengenerierung dar (vgl. Habann 1999, 23 ff.).

Die Ressourcenfrage ist also in mehrfacher Hinsicht eng mit der Ökonomisierung verbunden. Erstens sind allokative Ressourcen, die zur Medienproduktion notwendig sind, nur bei anhaltendem Unternehmenserfolg in ausreichendem Maße vorhanden. Für den Unternehmenserfolg hat die Marktorientierung eine Schlüsselrolle, so dass über die Ressourcen Unternehmensentscheidungen und Marktverhalten gekoppelt werden. 
Zweitens sollen Ressourcen Nutzen stiften, also vor allem Kosten- oder Leistungsvorteile erbringen. Beobachtbar ist auch im Medienbereich eine Ökonomisierung der Ressourcenallokation, bei der die Frage in den Vordergrund rückt, wie Ressourcen effektiv gesteuert und verteilt werden können, um das Prinzip der Kostenminimierung zu verfolgen.

Im Zuge dieser Entwicklung werden das Ressourcen- und das Verfahrensmanagement auf die ökonomischen Herausforderungen abgestimmt (vgl. Altmeppen 2000a). Die Anforderungen an das Ressourcenmanagement bestehen darin, die Budgets, die sich aus der Differenz von Kosten und Erlösen ergeben, zu planen, zu steuern und zu verteilen. Dementsprechend entscheidet das Ressourcenmanagement zum Beispiel darüber, welche Budgets für die Produktionsprozesse und welche für produktbegleitende Prozesse (Werbung, Marketing) zur Verfügung stehen. Insbesondere die Werbung avanciert derzeit zu einer wichtigen Ressource, denn mittlerweile sind die Medien selbst ihr bester Werbekunde (vgl. Horizont 43/2000, 26.10.2000, 6).

In engem Zusammenhang mit dem Ressourcenmanagement steht das Verfahrensmanagement, mit dem die Entscheidungsprogramme koordiniert und die Ressourcen zielgerecht eingesetzt werden. Vom Verfahrensmanagement sind in erster Linie die Produktionsprozesse betroffen, die Koppelung von Verfahrens- und Ressourcenmanagement zeigt sich am deutlichsten am Beispiel des Outsourcing. Dies erfordert ein anderes Verfahrensmanagement als wenn die Medienunternehmen inhouse produzieren. Beim Süddeutschen Verlag gibt es eine „konsequente Vergabe von nicht zum Kerngeschäft gehörenden Tätigkeiten an externe Stellen und eine extrem schlanke interne Organisation." (Schreiber 1999, 246) Die Entscheidung zum Outsourcing bedeutet ein verändertes Verfahrensmanagement, also jener organisationsinternen Handlungspraktiken, mit denen die Entscheidungsprogramme (Planung und Kontrolle der Produktionsprozesse, des Marketings und des Personal- und Qualitätsmanagements) umgesetzt werden. Mit Outsourcing sind andere Formen der Arbeitsgestaltung, der Arbeitsverträge und der Produktkontrolle verbunden als wenn inhäusig produziert wird. Outsourcing beispielsweise erfordert höhere Transaktionskosten (Suche nach geeigneten Produzenten und Inhalten, Gewährleistung der Produktion, Qualitätskontrolle) als die interne Produktion, es kann aber langfristig - aufgrund der geringeren Fixkosten und der Möglichkeit, Verträge schneller aufzulösen, - die kostengünstigere Produktionsart sein (vgl. Heinrich 1999, 157 ff.).

\section{6. Ökonomisierung des Angebotes}

Da die Medienunternehmen fortwährend das Dreieck von Budget, Quote und Inhalt austarieren müssen, wird - vor allem unter den Bedingungen hoher Fixkosten, dem Zwang zu innovativem Unternehmensverhalten sowie der nur sehr bedingt bestimmbaren Publikumsattraktivität (vgl. Kiefer 2001, 190f.) - die Vermarktung immer wichtiger. Damit verbunden ist ein weiterer Ökonomisierungsdruck im Hinblick auf das Angebot: Die Medienunternehmen besinnen sich immer weniger auf die Stärkung der publizistischen Dienstleistung, sondern vermehrt auf eine (absatz-)marktgerechte Produktgestaltung.

Das betrifft zum einen die Medienangebote selbst, die mittels immer feinerer Methoden der Marktforschung eingeführt werden. Insbesondere Unterhaltungs- und Infotainmentprodukte werden vor der Ausstrahlung vielfach getestet: Die Stoffentwicklung von der Idee zum fertigen Drehbuch, das Casting von Darstellern und Moderatoren, der Sendeplatz und die begleitenden Marketingaktivitäten werden empirisch 
und vor allem im Hinblick auf den Markterfolg evaluiert (vgl. Schmidt/Spieß 1997, 43 ff.; Siegert 2000, 187 ff.).

Die Ergebnisse der Markt- und Meinungsforschung schlagen ebenso wie die zunehmenden Markenbildungsprozesse unmittelbar bis auf die Produktionsprozesse durch und verändern die Veröffentlichungsentscheidungen. Bei den unterhaltungsorientierten Medienangeboten wirken sich vor allem die Markenbildungsprozesse aus, durch die Idee und Umsetzung von Medienprodukten den Strategien der Markenbildung und des Marketings unterworfen werden.

Informationsorientierte Medienangebote werden zunehmend weniger nach publizistischen Kriterien ausgewählt und produziert. Die Programmplanung richtet sich nach vermeintlichen oder tatsächlichen Absatzchancen, redaktionelle Freiheiten werden beschnitten, da die Produkte nach Vermarktungs- und Wertschöpfungsgesichtspunkten ausgewählt werden (vgl. Altmeppen 2000). Mit den Schlagworten vom Redaktionsmanagement und -marketing soll der „Markterfolg auf dem Wege des konzeptionellen, organisatorischen Personal- und Kostenmanagements“ gesichert werden (Meckel 1999, 22). Im Marketing wird die Marktorientierung von Medienunternehmen - und damit die Anfälligkeit für organisationsinterne Ökonomisierung - in deutlicher Weise erkennbar.

Aufgrund der Entscheidungen und Strategien, der Ressourcen und Verfahren der Medienunternehmen wird, so kann aus den bisherigen Thesen geschlossen werden, das publizistische Handeln durch wirtschaftliches Handeln überformt. Beschleunigt wird dieser Prozess der Ökonomisierung, weil die jeweiligen Medienangebote sich auf den Märkten bewähren müssen. Der Markt ist ein öffentlicher Ort, auf dem der Absatz von Produkten in Form der Akzeptanz des Publikums beobachtet werden kann. Doch nicht nur die Quoten und Auflagen, sondern auch die dahinter stehenden Unternehmensentscheidungen werden sichtbar und können von den Konkurrenten beobachtet werden. Mit der Etablierung neuer Programmformate und neuer Zeitschriftentitel (wie etwa dem Segment der Wirtschaftszeitungen) und ihrer Akzeptanz durch die Rezipienten werden neue Marktstrukturen geformt. Die Konkurrenz reagiert auf die beobachteten Prozesse und die neuen Strukturen wiederum mit eigenen Strategien, indem erfolgreiche Produktlinien imitiert oder eigene innovative Konzepte umgesetzt werden.

\section{7. Ökonomisierung der Ökonomisierung}

Mit der rekursiven Regulierung und Strukturierung kann eine Spirale beschrieben werden, bei der Strategien und Strukturen der Unternehmen und die Strukturen der Märkte sich gegenseitig beeinflussen und vorantreiben. Diese Spirale macht zudem deutlich, dass die Ökonomisierung keinesfalls allein dem Wettbewerb zugeschrieben werden kann, sondern dass die Medienunternehmen aktiv an der Ökonomisierung beteiligt sind. Dieser Prozess kann als Ökonomisierung der Ökonomisierung definiert werden. Jeder Schritt eines Unternehmens hin zu vermehrter Marktorientierung wird von den Wettbewerbern mit konkurrierenden ökonomischen Strategien beantwortet. Der jahrelange Prozess hin zur TV-Marktbeherrschung durch die Senderfamilien Kirch und Bertelsmann ist ein ausdrucksstarker Beleg für rekursive Interdependenzen und Anpassungsverhalten zwischen dem strategischen Handeln und den Strukturen der Unternehmen und den Märkten und dem aktiven Beitrag der Unternehmen zur Ökonomisierung.

Ökonomisierung wird also „produziert“, sie vollzieht sich keineswegs als Resultat unbeeinflussbarer Marktkräfte, ganz im Gegenteil wirken die Medienunternehmen erheblich an der Ökonomisierung mit. Der Grad des unternehmerischen Einflusses hängt davon ab, welche Gestaltungsmacht die Medienunternehmen mobilisieren können. 
Gestaltungsmacht als Faktor der Ökonomisierung speist sich aus den zwei Komponenten Verhandlungsmacht und Kapitalmacht. Die Formen beider Komponenten gehen zurück auf die Ressourcen der Medienunternehmen. So entsteht Verhandlungsmacht im Zusammenhang mit autoritativen Ressourcen. Diese „beziehen sich auf Typen des Vermögens zur Umgestaltung, die Herrschaft über Personen oder Akteure generieren" (Giddens 1997, 86) und äußern sich beispielsweise in Reputation, Image und schließlich im Einfluss. Mit Verhandlungsmacht suchen Medienunternehmen beispielsweise politischen Einfluss geltend zu machen, unter anderem, um die De-Regulierung von Medienmärkten voranzutreiben. Auch im ökonomischen System unterstützt die Verhandlungsmacht erfolgreiche Geschäftsabschlüsse, zum Beispiel bei anstehenden Fusionen.

Das Ausmaß der Verhandlungsmacht hängt in starkem Maße von der Kapitalmacht der Unternehmen ab. Kapitalmacht entsteht aus der Verfügbarkeit allokativer Ressourcen, also Formen des Vermögens zur Umgestaltung und der „Herrschaft über Objekte, Güter oder materielle Phänomene“ (Giddens 1997, 86). Mit der Verbindung von Verhandlungs- und Kapitalmacht erwachsen den Medienunternehmen Möglichkeiten, die Regulierung und Strukturierung der Wettbewerbsverhältnisse und die Marktergebnisse in ihrem Sinne zu gestalten.

Am Beispiel Multimedia und Bertelsmann wird erkennbar, wie sich mediale Gestaltungsmacht entwickelt und wie diese Macht auf rekursiven Regulierungen und Strukturierungen beruht. In dem Moment, wo Bertelsmann Multimedia als Zukunftsmarkt entdeckte, wurden zwei Entwicklungsstufen zu zentralen Entscheidungsprämissen des Konzerns: die zentrale Koordination der Multimedia-Aktivitäten und die Schaffung einer Produktlinie Multimedia (vgl. Liedl 1999). Diesen Entscheidungsprämissen folgt nun die Umsetzung über Entscheidungsprogramme in den einzelnen Unternehmensbereichen - beim Verfahrensmanagement beispielsweise durch umfassendes externes Wachstum (Aufkauf von oder die Beteiligung an einer Vielzahl von Unternehmen im Bereich Multimedia) sowie unternehmensintern durch die Einrichtung eines neuen Unternehmensbereiches „Multimedia“ sowie die Ausrichtung bestehender Bereiche wie Buchklub auf multimediale Aktivitäten. Zugleich wurde mit dem Rückzug von Bertelsmann aus dem Pay-TV-Geschäft und der Beendigung der Allianz mit Kirch das Marktverhalten verändert. Mit diesen Aktivitäten versucht Bertelsmann - ganz im Sinne einer aktiven, rekursiven Regulierung und Strukturierung - den Markt Multimedia, der derzeit überhaupt erst einmal geschaffen werden muss, im Sinne der eigenen Strategien (mit) zu gestalten.

\section{Resümee}

Mit organisationssoziologischen Studien kann der zentrale Raum der Koordinationsund Entscheidungsprozesse in den Medienunternehmen erfasst und seine Verschränkung mit anderen Unternehmen und Märkten thematisiert werden. Die medienunternehmerischen Strategien und die Marktstrukturen stehen in einem Verhältnis rekursiver Regulierung und Strukturierung, was erheblich zur Ökonomisierung beiträgt. Die Strategien der Medienunternehmen beeinflussen die Marktstrukturen, wie umgekehrt die Marktstrukturen sich auf die Strategien der Unternehmen auswirken. Mit diesen Verschränkungen wird die Ökonomisierung der Medien spiralförmig vorangetrieben.

In die Prozesse der Ökonomisierung sind die Medienunternehmen aktiv eingebunden. Durch Verhandlungs- und Kapitalmacht suchen sie nach Möglichkeiten, Märkte und Wettbewerbsbedingungen in ihrem Sinne zu gestalten. Der aktive Part der Me- 
dienunternehmen an der Ökonomisierung ist nicht die einzige Dimension, aber zumindest doch eine treibende Kraft der Ökonomisierung.

\section{Literatur}

Altmeppen, Klaus-Dieter (1996): Märkte der Medienkommunikation. Publizistische und ökonomische Aspekte von Medienmärkten und Markthandeln, in: ders. (Hg.): Ökonomie der Medien und des Mediensystems. Grundlagen, Ergebnisse und Perspektiven medienökonomischer Forschung, Opladen, S. $251-272$.

Altmeppen, Klaus-Dieter (1999): Redaktionen als Koordinationszentren. Beobachtungen journalistischen Handelns, Opladen.

Altmeppen, Klaus-Dieter (2000): Funktionale Autonomie und organisationale Abhängigkeit. Inter-Relationen von Journalismus und Ökonomie, in: Martin Löffelholz (Hg.): Theorien des Journalismus. Ein diskursives Handbuch, Opladen, S. 225 - 239.

Altmeppen, Klaus-Dieter (2000a): Medienmanagement als Redaktions- und Produktionsmanagement, in: Matthias Karmasin/Carsten Winter (Hg.): Grundlagen des Medienmanagements, München, S. $41-58$.

Altmeppen, Klaus-Dieter (2001): Gestaltungsmacht durch medienwirtschaftliches Handeln. Zur Rekursivität von Unternehmensstrategien und Marktstrukturen, in: Matthias Karmasin/Manfred Knoche/Carsten Winter (Hg.): Medienwirtschaft und Gesellschaft, Bd. 1: Medienunternehmen \& Medienproduktion (im Druck).

Altmeppen, Klaus-Dieter/Patrick Donges/Kerstin Engels (1999): Transformation im Journalismus. Journalistische Qualifikationen im privaten Rundfunk am Beispiel norddeutscher Sender, Berlin.

Giddens, Anthony (1997): Die Konstitution der Gesellschaft. Grundzüge einer Theorie der Strukturierung, 3. Aufl., Frankfurt a. M./New York.

Habann, Frank (1999): Kernressourcenmanagement in Medienunternehmen, Lohmar/Köln.

Heinrich, Jürgen (1999): Medienökonomie. Bd. 2: Hörfunk und Fernsehen, Opladen/Wiesbaden.

Hill, Wilhelm/Raymond Fehlbaum/Peter Ulrich (1994): Organisationslehre. Ziele, Instrumente und Bedingungen der Organisation sozialer Systeme, 5. überarb. Aufl., Bern/Stuttgart/Wien.

Horizont, Zeitung für Marketing, Werbung und Medien, Frankfurt a. M.

Kiefer, Marie Luise (2001): Medienökonomik. Einführung in eine ökonomische Theorie der Medien, München/Wien.

Krüger, Wilfried (1994): Organisation der Unternehmung, 3. verb. Aufl., Stuttgart/Berlin/Köln.

Liedl, Reinhard (1999): Strategien und Aktivitäten von Bertelsmann im Multimediabereich, in: Matthias Schumann/Thomas Hess (Hg): Medienunternehmen im digitalen Zeitalter. Neue Technologien - neue Märkte - neue Geschäftsansätze, Wiesbaden, S. 203 - 218.

Luhmann, Niklas (1987): Soziale Systeme. Grundriß einer allgemeinen Theorie, Frankfurt a. M.

McQuail, Dennis (1986): Kommerz und Kommunikationstheorie, in: Media Perspektiven (10), S. $633-643$.

Meckel, Miriam (1999): Redaktionsmanagement. Ansätze aus Theorie und Praxis, Opladen/Wiesbaden.

Moss, Christoph (1998): Die Organisation der Zeitungsredaktion: wie sich journalistische Arbeit effizient koordinieren läßt, Opladen/Wiesbaden.

Ortmann, Günther/Jörg Sydow/Arnold Windeler (1997): Organisation als reflexive Strukturation, in: Günter Ortmann/Jörg Sydow/Klaus Türk (Hg.): Theorien der Organisation: die Rückkehr der Gesellschaft, Opladen, S. 315 - 354.

Röper, Horst (2001): Formationen deutscher Medienmultis 1999/2000: Entwicklungen und Strategien der größten deutschen Medienunternehmen, in: Media Perspektiven (1), S. 2 - 30.

Schmidt, Siegfried/Brigitte Spieß (1997): Die Kommerzialisierung der Kommunikation. Fernsehwerbung und sozialer Wandel 1956-1989, Frankfurt a. M.

Schreiber, Gerhard Andreas (1999): New Media im Süddeutschen Verlag, in: Matthias Schumann/Thomas Hess (Hg): Medienunternehmen im digitalen Zeitalter. Neue Technologien neue Märkte - neue Geschäftsansätze, Wiesbaden, S. 233 - 247. 
Siegert, Gabriele (2000): Medienmanagement als Marketingmanagement, in: Matthias Karmasin/Carsten Winter (Hg.): Grundlagen des Medienmanagements, München, S. 173 - 195.

Sjurts, Insa (1996): Die deutsche Medienbranche: eine unternehmensstrategische Analyse, Wiesbaden.

von der Oelsnitz, Dietrich (2000): Marktorientierte Organisationsgestaltung. Eine Einführung, Stuttgart/Berlin/Köln.

Weischenberg, Siegfried (1992): Journalistik. Theorie und Praxis aktueller Medienkommunikation, Bd. 1: Mediensysteme, Medienethik, Medieninstitutionen, Opladen.

Zimmer, Marco/Günther Ortmann (1996): Strategisches Management, strukturationstheoretisch betrachtet, in: Hans H. Hinterhuber/Sayad Al-Ani/Gernot Handlbauer (Hg.): Das neue strategische Management: Elemente und Perspektiven einer zukunftsorientierten Unternehmensführung, Wiesbaden, S. 87-114. 